\title{
Towards Automated Tracking of Initiation and Propagation of Cracks in Aluminium Alloy Coupons Using Thermoelastic Stress Analysis
}

\author{
C. A. Middleton ${ }^{1}$ (D) A. Gaio ${ }^{1,3} \cdot$ R. J. Greene ${ }^{2}$ E. A. Patterson ${ }^{1}$
}

Received: 3 June 2018 / Accepted: 17 December 2018 / Published online: 12 January 2019

(c) The Author(s) 2019

\begin{abstract}
Full-field non-destructive evaluation techniques are useful tools for indicating damage in fatigue-prone environments. Here, the full-field technique thermoelastic stress analysis (TSA) is employed to locate and track naturally initiating cracks in aluminium alloy specimens. An algorithm, based on the concept of optical flow, is used to track changes in the characteristic stress field present ahead of a crack during loading, and thereby to locate the crack-tip position. The initiation of cracks is indicated at sub-mm lengths, before the crack is observable by visual inspection, and in bolted specimens TSA indicates the presence of a crack before it extends beyond the bolt-head. The crack path propagation is mapped and matches well with the final crack geometry. Results are equivalent for surfaces prepared with matt black and aircraft primer paint, showing that optical flow processing of TSA data would be particularly useful for crack tracking in aerospace applications, as no additional surface preparation would be required to implement full-field TSA measurements.
\end{abstract}

Keywords TSA $\cdot$ Optical flow $\cdot$ Crack-tip tracking $\cdot$ Fatigue

\section{Introduction}

The development of fatigue cracks is a problem in a wide range of industrial contexts. Therefore, non-destructive methods to detect the initiation and propagation of these cracks are necessary in order to allow monitoring and remedial action to be undertaken. This monitoring must often be undertaken in areas which are difficult to access, or during service [1]. Methods which are currently applied include visual inspection, placement of strain gauges, magnetic particle testing, ultrasound, digital image correlation (DIC), and

Electronic supplementary material The online version of this article (https://doi.org/10.1007/s10921-018-0555-4) contains supplementary material, which is available to authorized users.

C. A. Middleton

ceri.middleton@liverpool.ac.uk

1 School of Engineering, University of Liverpool, The Quadrangle, Brownlow Hill, Liverpool L69 3GH, UK

2 Strain Solutions Ltd,

Dunston Innovation Centre, Dunston Road, Chesterfield, Derbyshire S41 8NG, UK

3 Present Address: Additive Manufacturing Technologies Ltd, Alison Business Centre, 39/40 Alison Crescent, Sheffield S2 1AS, UK electronic speckle pattern interferometry (ESPI) [1-3]. Each of these have drawbacks, for example, strain gauge measurements require prior knowledge of expected crack sites as their use only allows localised data collection. Visual inspection relies on the ability of an inspector to locate damage, so it is both time consuming and labour intensive to monitor or inspect large structures.

Full-field methods, such as DIC or ESPI, remove the need for prior knowledge of potential crack sites as they can be used to observe larger areas. However, the complicated surface preparation (DIC) or complicated equipment (ESPI) required do not make these techniques ideal for inspections on a short timescale, or for in-service monitoring [4]. Thermoelastic stress analysis (TSA) is an alternative full-field method which requires only that the surface has a uniform high emissivity. For metallic materials, this necessitates minimal surface preparation; namely, a layer of matt paint [5-7]. The quality of data returned by TSA compared favourably in a comparison of these three full-field techniques in a study by Rajic et al. [8].

TSA maps the surface stresses in a material based on small temperature variations measured by an infrared (IR) detector while the material is dynamically loaded [5,9]. Cracks or damage can therefore be located due to the indicative stresses present, which has been exploited previously in a range of 
studies [10-15]. The position of the crack is often determined from the phase profile along the line of the crack, which requires knowledge of the crack location and orientation [10-12]. An alternative approach, using the second harmonic of the loading frequency, also allows the plastic zone associated with damage, or the damage itself, to be located $[16,17]$.

Previous work has also shown that TSA is very sensitive to crack initiation; locating microcracks which were only observed visually ex situ by analysis of thin sections [18]. Advances in technology, including microbolometers, also mean that TSA is becoming a cheaper and more flexible option than previously understood [19, 20].

The automation of crack location procedures using TSA is an interesting topic of development, as it would increase the potential applications in industrial settings. Ancona et al. [11] have automated the location of cracks during fatigue loading assuming prior knowledge of the initial crack position, whilst Rajic and Brooks [12] have tracked cracks in real time, combining a TSA system with a $\mathrm{X}-\mathrm{Y}$ movement stage to follow the propagation of a crack. Both these automated methods involve tracking the propagation of artificially induced cracks, where the crack-tip stresses were well-developed before observations began. However, it is also of interest to locate cracks when they initiate naturally over time, and then track their propagation with no prior knowledge of the crack location or direction of growth.

Here, the initiation and propagation of naturally-initiated cracks are monitored using TSA during tension-tension fatigue loading of metallic 'simple' specimens and 'complex' double-lap-joints under laboratory conditions. Postprocessing of the data is carried out using an optical flow method which indicates the initiation of a crack, and then tracks its propagation, allowing the crack path to be mapped.

\section{Methods}

\subsection{Specimens}

Two sets of specimens were machined from aluminium alloy. 'Simple' quasi two-dimensional coupons of seven different geometries (Fig. 1) were manufactured from a single sheet of aluminium alloy 2024-T3, of thickness $1.6 \mathrm{~mm}$. The simple specimens were designed so that stress concentrations would occur during cyclic loading, leading to crack initiation. However, the exact position of crack initiation was unknown before loading began. The specimens were painted with matt black paint (Plasti-kote 23101 Premium Spray Paint, matt black).

'Complex' double-bolted, double-lap joint specimens were also designed, with specimen dimensions based on a combination of standards [21, 22] (Fig. 2). External plates were machined from the same sheet of aluminium alloy 2024T3 as the simple specimens and internal plates from one sheet of aluminium alloy 6082-T6. The holes in one external plate were slightly larger, promoting crack initiation on the observed side of the specimen, and hence simplifying the experimental procedure. In order to maintain the distance between the external plates and avoid bending due to the pressure of the load frame grips, a spacer of the same thickness as the internal plate was positioned between the external plates at the end furthest from the bolts and attached with a cyanoacrylate adhesive. The external plate to be monitored was painted with the same matt black paint used for the simple specimens. Cadmium-plated steel bolts (UNF AN4-5A; LAS Aerospace Ltd, Devon, $U K$ ) and appropriate nuts and washers were positioned such that the head of the bolt was located on the external plate with the larger hole, and tightened to a torque of $23 \mathrm{Nm}$. The bolts were then painted with matt black paint, with careful attention being paid to not disturb the paint on the plates. After assembly, standard aircraft sealant was applied around the bolt-heads of one specimen.

\subsection{Experimental Methodology}

Specimens were cyclically loaded in an Instron 8501 universal testing machine (Instron, Buckinghamshire, UK) until crack development and often complete failure. Loading was sinusoidal with maximum and minimum loads $\left(F_{\max }\right.$ and $F_{\text {min }}$ respectively) chosen such that $R\left(F_{\text {min }} / F_{\text {max }}\right)=0.1$. Due to the different geometries, the load values required to generate crack growth on a reasonable timescale were different between specimens, and are detailed in Table 1.

During loading, image data were collected at intervals ranging from 250 to 1000 cycles and processed to stress data using a DeltaTherm 1780 system (Stress Photonics, Madison, WI), which combines a FLIR SC7650 cooled infrared camera with DeltaTherm TSA processing software. Processing of each dataset took approximately $10 \mathrm{~s}$. The theory of TSA has been described elsewhere [5] and here only analysis of the signal magnitude (thermoelastic response) data is reported. For the bolted specimens, TSA data were collected from the face of the specimen with the bolt-head. Visible light images were obtained at the same time as TSA data for one specimen, for this experiment the visible light camera was positioned perpendicular to the specimen, with the infrared camera at a slight angle to avoid conflicting fields of view.

\subsection{Crack Location}

A variety of methods has been used previously to indicate the location of a crack in TSA data [10-12, 23]. The stress distribution ahead of a crack is represented in TSA signal magnitude data by a series of nested cardioid contours with a 

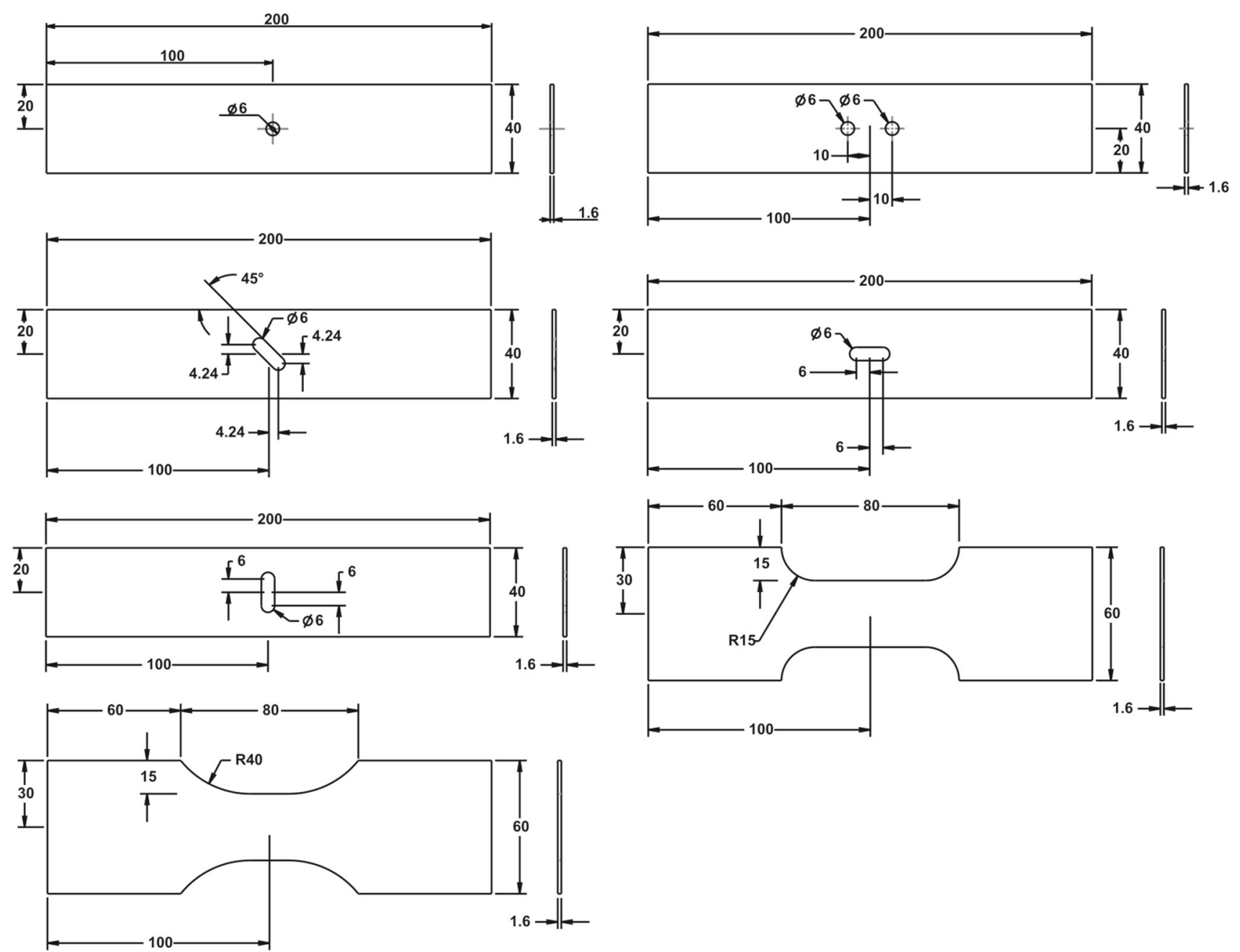

Fig. 1 Simple specimen geometries. Tension-tension loading was applied along the longitudinal axis of each specimen. Units in mm

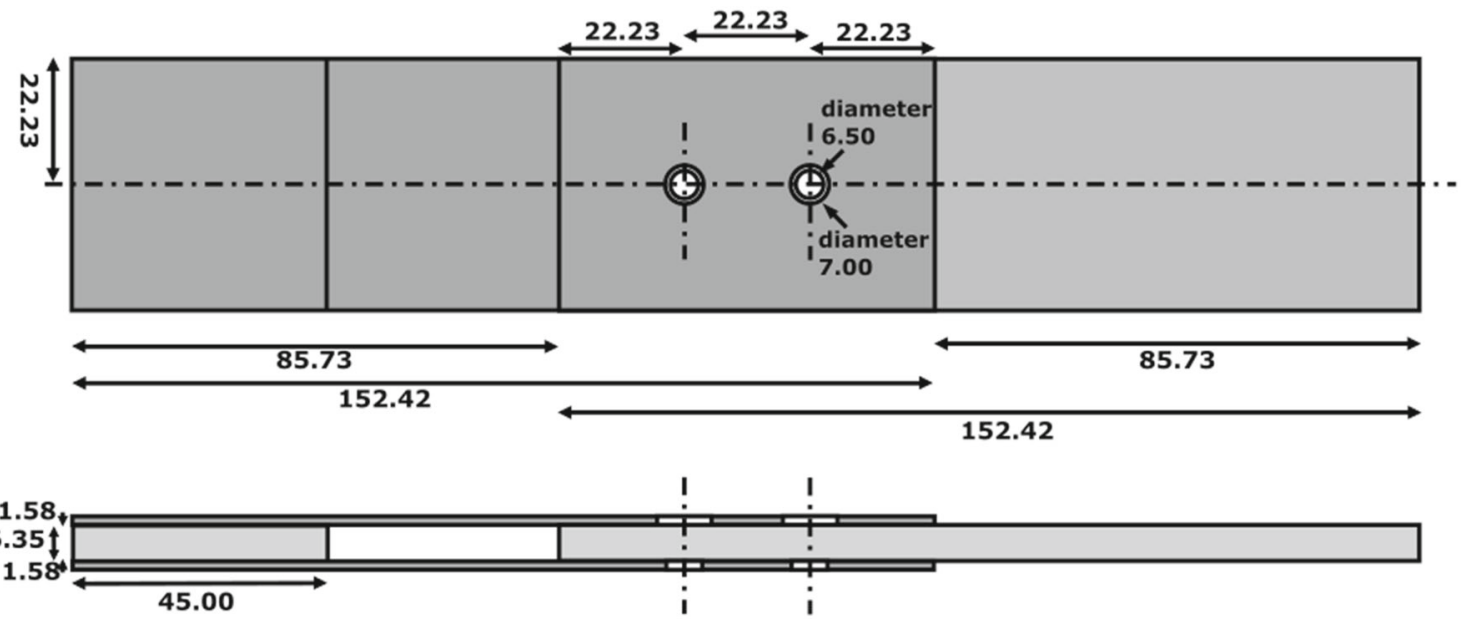

Fig. 2 Complex specimen geometry, plan and side view. Units in $\mathrm{mm}$

maximum value at the centre (Fig. 3; [10]). These indicative stress fields develop during initiation of a crack, and move as that crack propagates, therefore the crack-tip can be located by monitoring the position of its characteristic stress distribution. 
Table 1 Loading conditions and cycles to crack initiation and failure (or test end due to user imposed safety limits-indicative of imminent failure) for loaded specimens; where loading conditions were stepped, this is indicated in column 3 , with the number of cycles at each step shown in the right-hand column

\begin{tabular}{|c|c|c|c|c|c|c|c|}
\hline Specimen & Specimen geometry & Load condition & $F_{\max }(\mathrm{kN})$ & $F_{\text {min }}(\mathrm{kN})$ & Freq. $(\mathrm{Hz})$ & $\begin{array}{l}\text { Cycles to crack } \\
\text { initiation }\end{array}$ & $\begin{array}{l}\text { Cycles to load step, failure or } \\
\text { end of test }\end{array}$ \\
\hline 1 & One hole & & 9.6 & 0.96 & 19 & 124,000 & 140,000 (failure) \\
\hline $1 \mathrm{~b}$ & One hole, primer paint & & 9.6 & 0.96 & 19 & 65,500 & 85,000 (failure) \\
\hline 2 & Two holes & & 9.6 & 0.96 & 19 & 85,500 & 103,500 (failure) \\
\hline 3 & Diagonal slot & & 9.6 & 0.96 & 19 & 15,000 & 17,100 (failure) \\
\hline \multirow[t]{2}{*}{4} & \multirow[t]{2}{*}{ Vertical slot } & $\mathrm{a}$ & 14.06 & 1.406 & 19 & - & 20,000 \\
\hline & & $\mathrm{b}$ & 15.466 & 1.5466 & 19 & 6000 & 9600 (limits) \\
\hline 5 & Horizontal slot & & 8.64 & 0.864 & 19 & 18,750 & 20,700 (failure) \\
\hline 6 & Small radius side cut outs & & 11.62 & 1.162 & 13 & 62,500 & 64,600 (limits) \\
\hline \multirow[t]{4}{*}{7} & \multirow[t]{4}{*}{ Large radius side cut outs } & a & 11.62 & 1.162 & 13 & - & 10,000 \\
\hline & & $\mathrm{b}$ & 12.78 & 1.278 & 13 & - & 135,000 \\
\hline & & $\mathrm{c}$ & 14.06 & 1.406 & 13 & - & 150,000 \\
\hline & & $\mathrm{d}$ & 14.76 & 1.476 & 13 & 5250 & 6100 (limits) \\
\hline 8 & Bolted specimen & & 30.14 & 3.014 & 13 & 71,030 & 71,900 (failure) \\
\hline 9 & Bolted specimen with sealant & & 30.14 & 3.014 & 13 & 97,060 & 97,300 (failure) \\
\hline
\end{tabular}

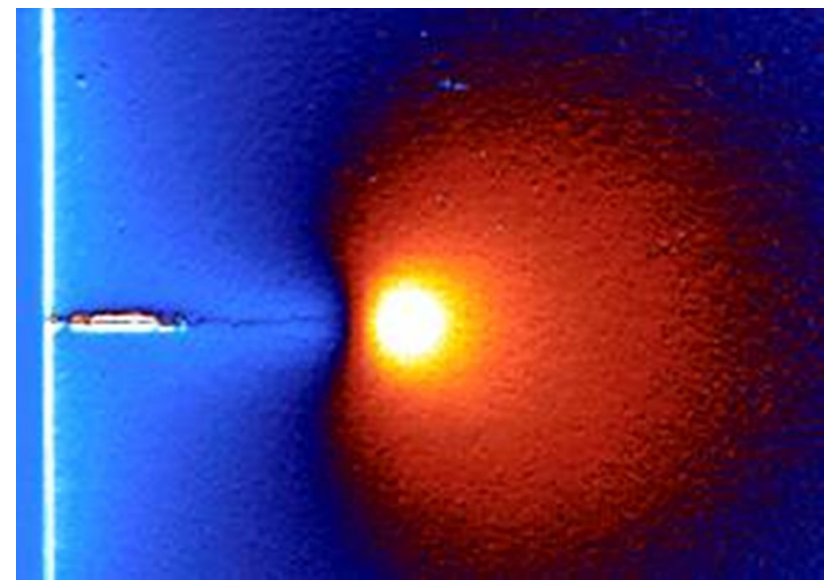

Fig. 3 Characteristic stress field ahead of a crack, propagating from left hand side. After Diaz et al. [10], with permission. Copyright John Wiley and Sons

The concept of optical flow is used here to track changes in the stress field, and hence the position of the crack-tip, by comparing subsequent TSA datasets during crack growth. As the stress peak advances in front of a propagating crack, a comparison of its position in two temporally-separated datasets allows determination of the crack-tip position at the time of the second dataset.

\subsection{Optical Flow Method}

Optical flow is used to represent the motion of "objects" over time in either two or three dimensions in a series of images. The velocity field calculated from a series of images allows the rate of change of the spatial arrangement of these objects to be determined [24]. Here, a simplified version of this technique is applied to data from two-dimensional TSA using the signal magnitude data, which is proportional to the first stress invariant, and the stress distribution associated with a crack is treated as the "object" to be tracked.

The key concept of the optical flow method is that, in the time domain, the stress distribution associated with the geometric features of the component are stationary whereas the stress distribution associated with a growing crack moves in the spatial domain. Hence, by identifying the changes with time in the stress field, it is possible to locate and track initiating and propagating cracks. The optical flow method is applied during post-processing of TSA signal magnitude data which is directly proportional to the first stress invariant. The TSA data fields are normalised using a value that is characteristic of the data field, for example the mean of the far-field, and subsequent fields are subtracted from one another, such that

$\Delta S(x, y, \Delta t)=S(x, y, t)-S(x, y, t+1)$

where $x$ and $y$ are spatial coordinates, $t$ is time and $S$ is the normalised measured amplitude of the thermoelastic signal. A typical difference field, and the TSA datasets from which it was calculated, are shown in Fig. 4 with no crack present. For the difference field, the standard deviation of the $\Delta \mathrm{S}$ value for each row and column are calculated, i.e. $\overline{\Delta S_{y}}(x)$ and $\overline{\Delta S_{x}}(y)$ respectively, where over-score indicates the standard deviation. In the absence of crack initiation or 

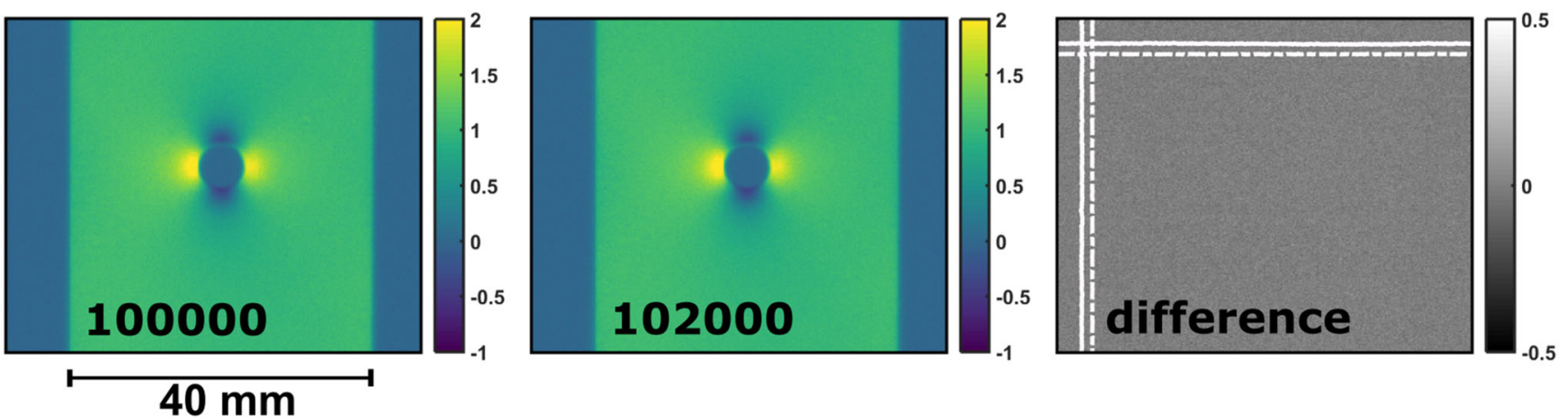

Fig. 4 Normalised TSA signal magnitude for given cycle numbers with no crack present, along with difference data calculated by the optical flow method. Lines correspond to mean (solid line) and standard deviation (dashed line) of columns and rows in the difference data

propagation, there will be no significant difference between the two data fields, i.e. $S(x, y, t) \approx S(x, y, t+1)$ and both $\overline{\Delta S_{y}}(x)$ and $\overline{\Delta S_{x}}(y)$ will be approximately constant with $x$ and $y$ respectively, as can be seen in Fig. 4. The process so far is repeated, retaining $S(x, y, t)$ but replacing $S$ $(x, y, t+1)$ by the next datafield in the time sequence. However, in the presence of an initiating or propagating crack then neither $\overline{\Delta S_{y}}(x)$ nor $\overline{\Delta S_{x}}(y)$ will be constant but both will show a significant excursion at the location of the stress distribution associated with the crack-tip, as shown in Fig. 5. The coordinates of the maximum excursions of $\overline{\Delta S_{y}}(x)$ and $\overline{\Delta S_{x}}(y)$ are taken as the location of the crack-tip $(x(t), y(t))$ and the process is repeated for the next timestep with the reference updated; $S(x, y, t+1) \stackrel{\text { updated }}{\longrightarrow} S(x, y, t)$. The size of a significant excursion in $\overline{\Delta S_{y}}(x)$ and $\overline{\Delta S_{x}}(y)$ can either be defined manually by the user or set to a given multiple of standard deviations in their values along $x$ and $y$ respectively.

In the absence of any cracks, the entire datafield can be processed using the optical flow method in the manner described above; however, once a crack has initiated and been located then a region of interest can be defined around and ahead of it. If the optical flow method is applied separately inside and outside of the region of interest, then the crack propagation can be tracked in the region of interest and, outside the region of interest, the initiation of an additional crack can be identified. In this study, once initiation of one crack was detected, only the region of interest was analysed, i.e. only the first crack to initiate was tracked.

\section{Results}

\subsection{Simple Specimens}

Tension-tension cyclic loading was carried out on the specimens with loading parameters as detailed in Table 1. For certain specimens, the load was increased in steps at the given cycle numbers, as no evidence of crack initiation had been observed in the real-time TSA data. Lower frequency loading was necessary at higher loads (e.g. Specimens 6 and 7) due to resonances in the equipment. In specimens at the same loading conditions, the number of load cycles required to naturally initiate a crack differed depending on the specimen geometry (Table 1; Specimens 1-3). Figure 5 shows the normalised TSA signal magnitude and difference data for specimen 1 (one circular hole) over the course of initiation and growth of fatigue cracks. Initiation occurred on the left-hand side of the hole earlier than the right-hand side, and here only the first appearing crack is tracked. The crack-tip positions are evident in the TSA data due to the peaks in signal magnitude ahead of the cracks, and in the difference data as areas of 'high' difference, shown by the peaks in the plotted curves. The optical flow method indicates a crack initiating at 124,000 cycles, which then propagated towards the left of the specimen, until 140,000 cycles, after which the specimen failed. A later crack also initiates on the right-hand side of the hole during growth of the tracked crack. Equivalent images for specimens 1b-7 are shown in the Supplementary Information.

Figure 6 shows the mapped crack-tip positions over time for each simple specimen, along with the crack length of the first crack which formed as a function of the cycle number. In all cracks, the growth accelerates as the crack length increases, as would be expected from classical fracture mechanics. For all specimens, the initial position of the crack is found within $1 \mathrm{~mm}$ of the edge of the specimen from which it initiates.

In most specimens, there is noise associated with the initial position of the crack found using the optical flow method during initiation, but this becomes much reduced as the crack grows. There is also an error in the crack-tip position for Specimen 5, which is most apparent in the crack length graph, where the crack appears to decrease in length. Possible explanations for the noise and the crack length irregularities will be discussed in Sect. 4. The lower number of data points for 

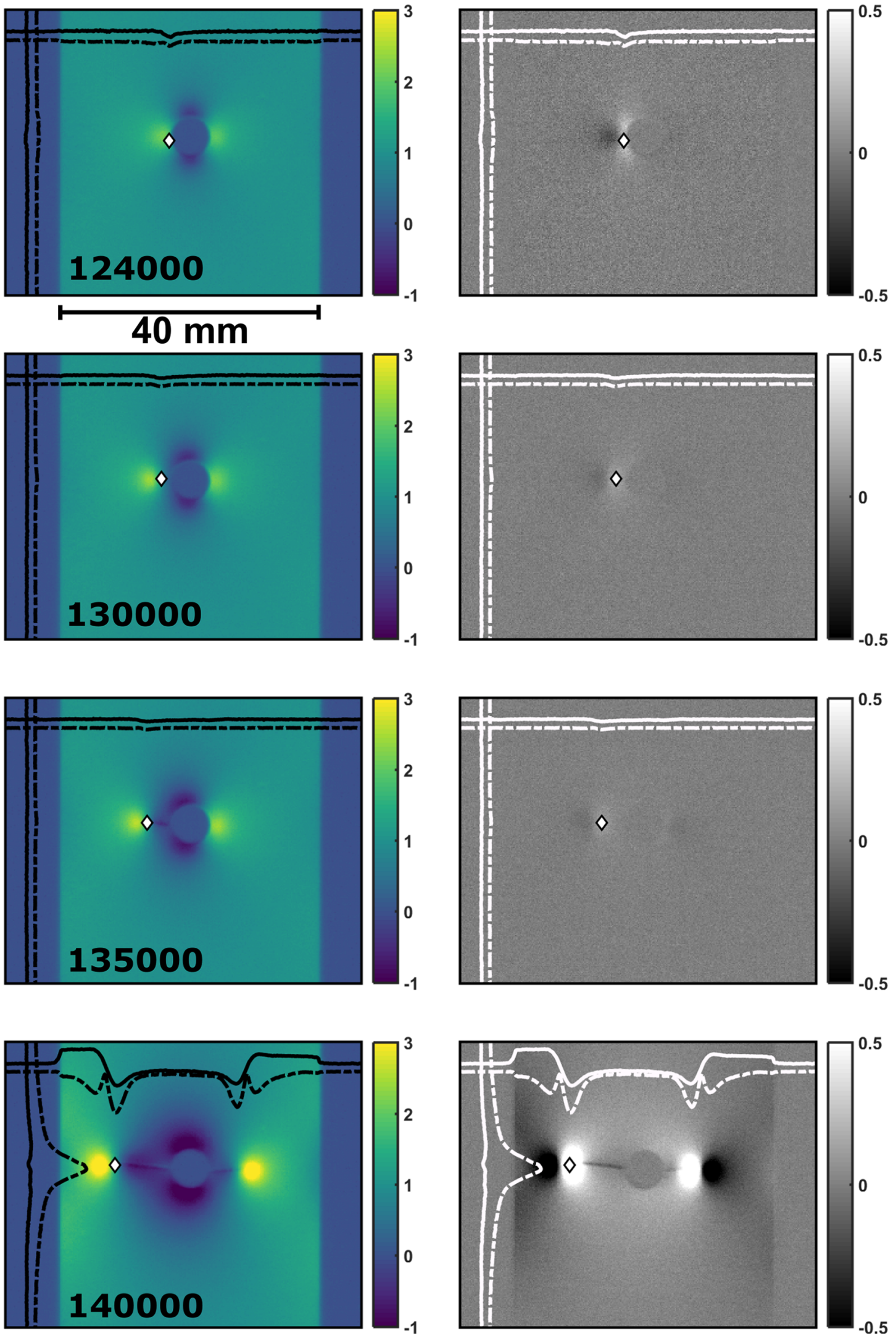

Fig. 5 Normalised TSA signal magnitude (left panels) and difference data (right panels) for the first appearing crack in specimen 1 (one circular hole) for given numbers of load cycles. Plots of the mean (solid

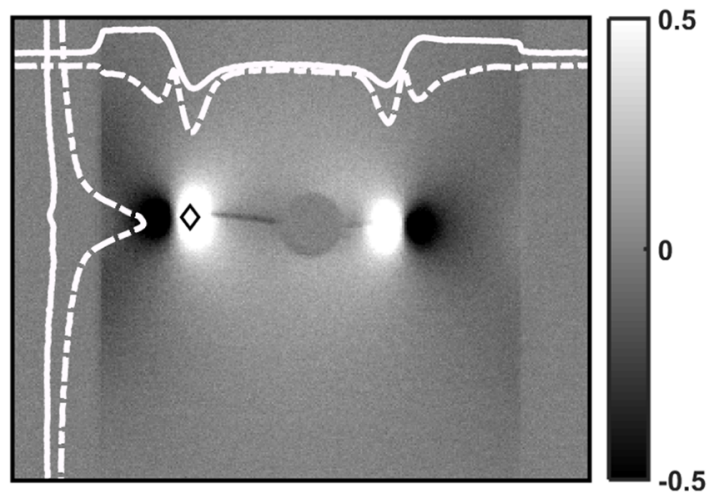

line) and standard deviation (dashed line) of the columns and rows of difference data are shown. Peaks in the standard deviation determine the position of the crack-tip (diamonds). Load axis is vertical 
(a)
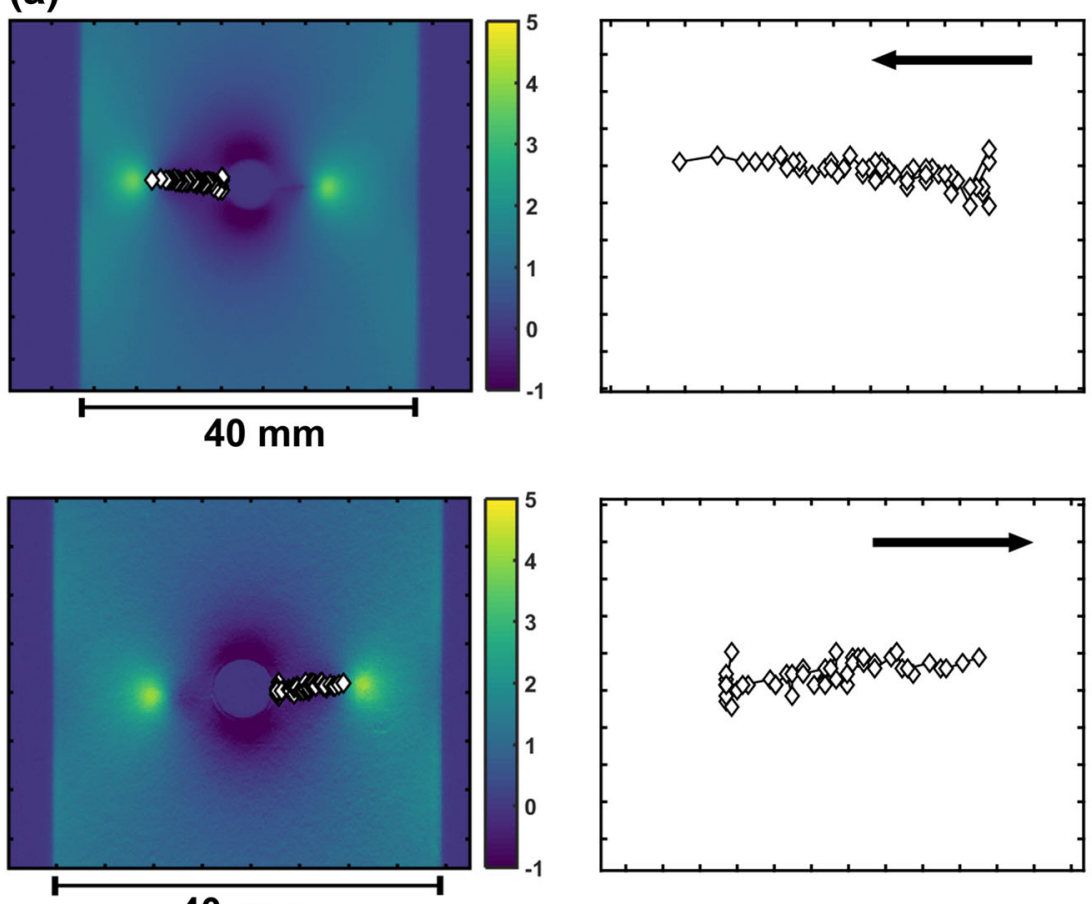

$40 \mathrm{~mm}$
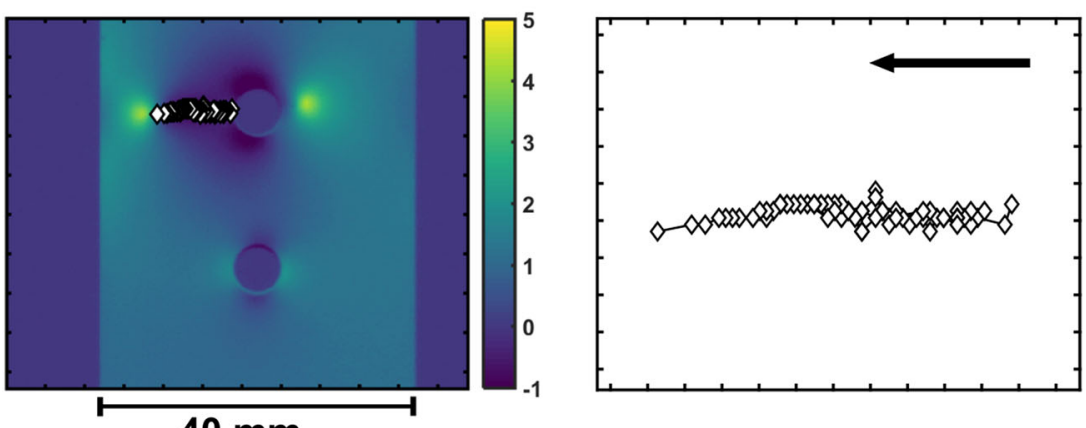

$40 \mathrm{~mm}$
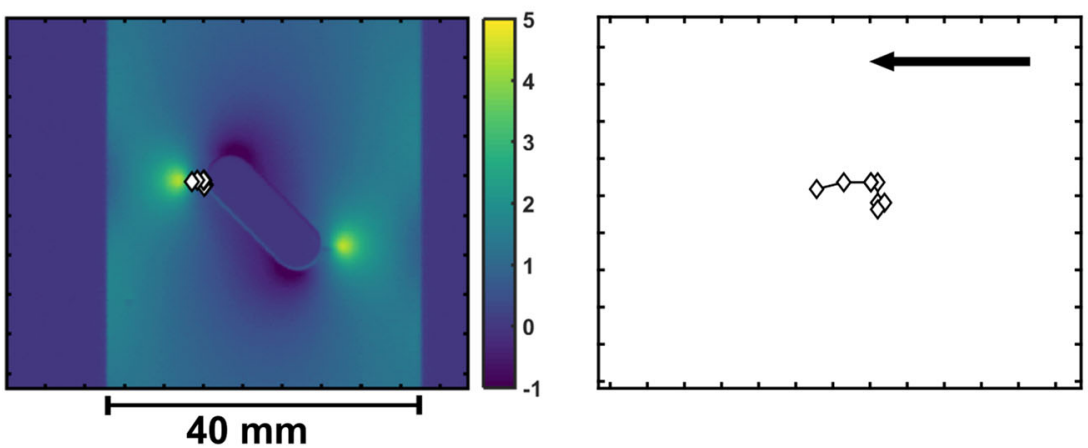

Fig. 6 Results of the optical flow method for a simple specimens 1, 1b (primer paint), 2 and 3 (from top), b simple specimens 4, 5, 6 and 7 (from top). Left panel shows the normalised TSA signal magnitude of the last dataset collected before failure, diamonds show the location of the crack-tips. The centre panel shows an enlargement of the location of
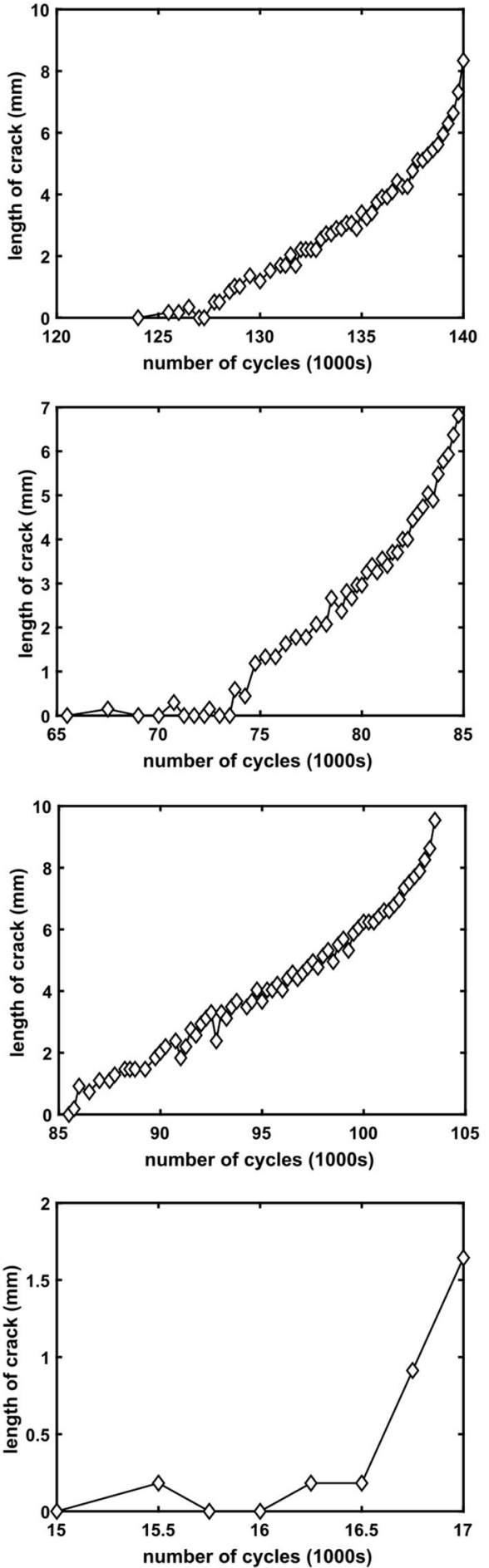

the first crack to appear, arrow indicates propagation direction. The right panel shows the length of the crack against the number of load cycles. Crack length presented here is the horizontal length with respect to the initial position found by the optical flow method. Tick marks in central panel at $1 \mathrm{~mm}$ intervals 
(b)
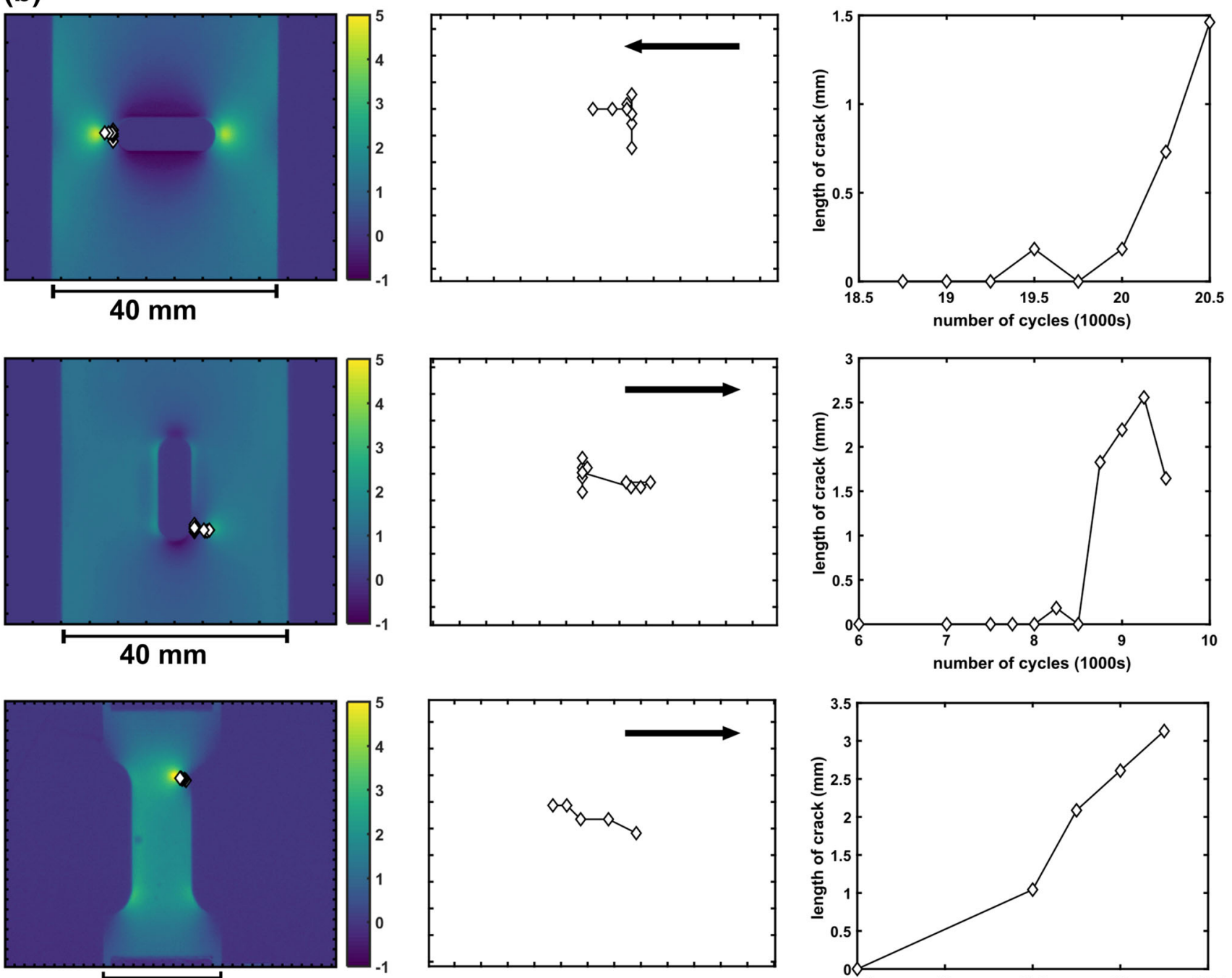

$60 \mathrm{~mm}$
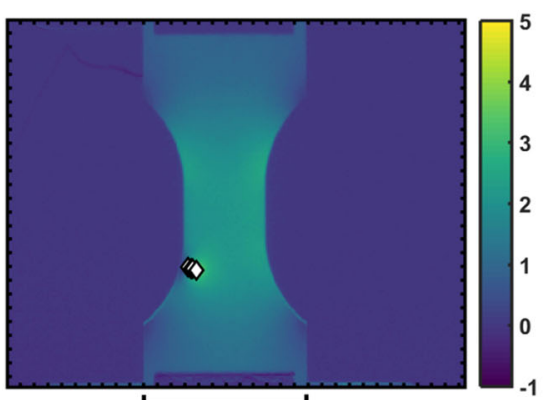

$60 \mathrm{~mm}$

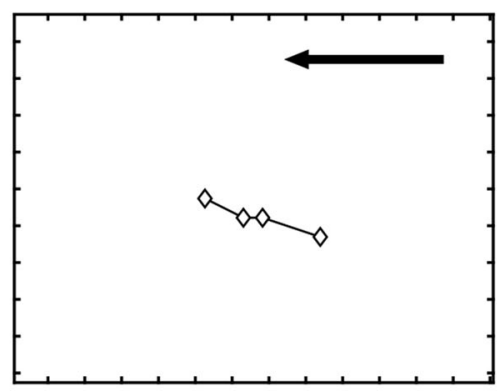

Fig. 6 continued

Specimens 3-7 compared to Specimens 1, $1 \mathrm{~b}$ and 2 are due to the faster crack growth-rate compared to the data collection rate at the given loading conditions.

A comparison of the TSA results from Specimens 1 and $1 \mathrm{~b}$ show that both the signal magnitude data and optical flow tracking are similar for specimens painted in either matt black or aircraft primer paint. A further comparison of these two specimens can be seen in Fig. 7, where the crack paths calculated with the optical flow method are compared to the final crack morphology. The positions located by the optical 

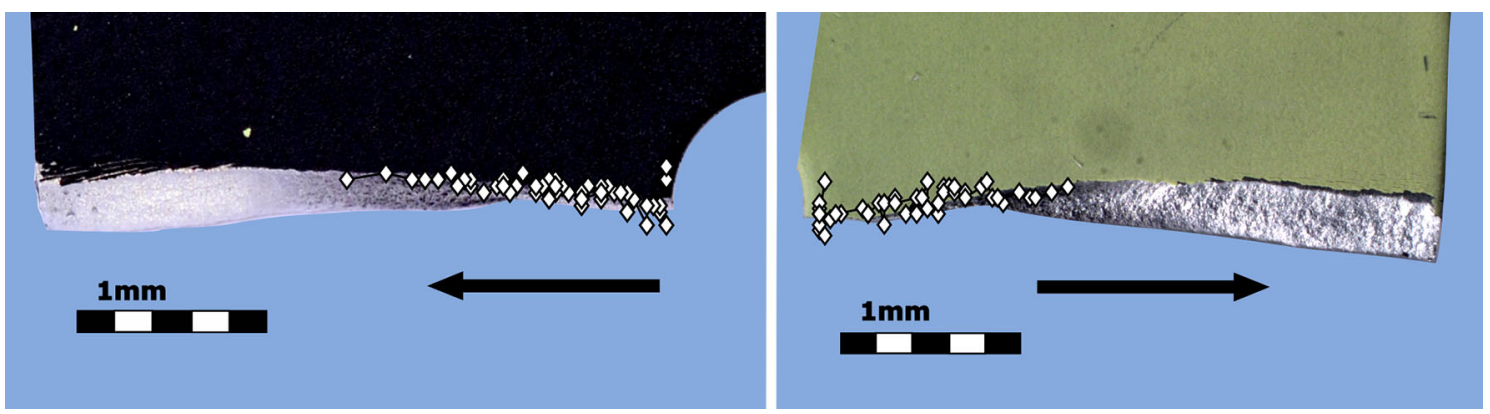

Fig. 7 Crack-tip position over time output from optical flow method (diamonds), overlain on final crack geometry for specimens with matt black paint coating (left hand side), and Airbus primer paint coating (right hand side). Arrows indicate crack growth direction, scales in mm

flow method are within $1 \mathrm{~mm}$ of the final crack path, with the accuracy increasing further from the initiation point, with longer cracks and at higher crack growth-rates.

\subsection{Bolted Specimens}

A comparison of the TSA signal magnitude data and visible light images collected during loading of the bolted specimen is shown in Fig. 8. Changes in the signal magnitude concentration, indicative of crack initiation, are evident in the TSA data (starting in cycle 70,120) before the crack is observable in the visible light images (cycle 70,770).

Processing of these datasets has been carried out with the optical flow method, the results of which are shown in Fig. 9. The crack is identified first on the right-hand side of the bolthead, and then tracked until failure. The optical flow method indicates the presence of a crack when the crack is difficult to observe in the magnified visible light images. The plots of standard deviation shown in Fig. 9 show increases associated with the bolts, not only the crack position. These changes are particularly evident at 71,030 cycles.

Figure 10 shows the TSA data collected from a specimen with sealant applied around the bolt-head. A similar change in signal magnitude distribution to that of the non-sealed bolted specimen is observed. The implementation of the optical flow method for this set of data results in a delay in identifying the initial location of the crack-tip position compared to the bolted specimen with no sealant. Once the characteristic crack-tip field appears from underneath the extent of the sealant, the crack can be tracked.

\section{Discussion}

As discussed in Sect. 1, TSA has previously been used to locate the position of cracks during fatigue loading [10-12]. Here, it has been shown that the stress distribution indicative of cracking is present in TSA data before a crack would be located by visual inspection, in particular when that crack has not yet emerged from under a bolt-head. An optical flow method has also been implemented which interrogates the TSA data to determine the crack-tip position. This method successfully indicates the initiation of a crack at sub-mm lengths in specimens with simple geometries and then maps the propagation of that crack until failure of the specimen. Overlays of the mapped crack position and the final morphology of two cracked specimens show that the optical flow method maps the shape of the cracks well (Fig. 7). For the one-hole specimen geometry, crack-tip positions are located along the full length of the crack, showing that tracking was possible throughout crack growth.

TSA data were collected at fixed cycle intervals, so in specimens where crack growth was fast relative to the data collection rate, the position was only tracked for a small distance. This effect was often due to fast failure of the specimen, so certain geometries show no coverage of the crack position after a few mm of growth. In particular, the high loading rate required to initiate cracking in specimens 6 and 7, also resulted in fast crack propagation. To mitigate the effects of crack growth-rate on the accuracy of crack location, the interval between TSA data collection can be decreased. In situ processing would determine whether each dataset is useful, avoiding the need to save large amounts of negligibly different data.

As can be seen in Fig. 7, the position indicated by the optical flow method does not exactly match the crack initiation point as seen in the final crack geometry. Similar noise in the initiation position can also be seen in the other specimens in Fig. 6. This noise generally decreases as the crack propagates and grows in length. It should be noted that although the initiation point in the simple specimens is not exact, the indicated initiation point is still within $\pm 1 \mathrm{~mm}$ of the final position perpendicular to the crack growth direction, and less parallel to the crack growth direction.

The lower accuracy of the optical flow method during initiation may be due to the way in which the characteristic stresses ahead of the crack evolve during crack initiation and propagation. During initiation, this stress distribution is not as well developed as when the crack is fully initiated. These effects can be seen in Fig. 5 where the peak normalised signal 

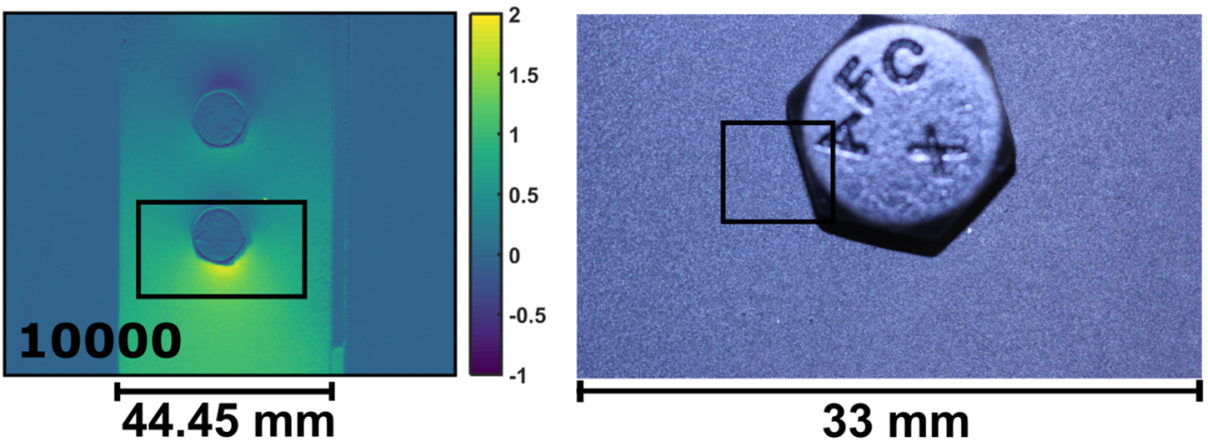

$33 \mathrm{~mm}$
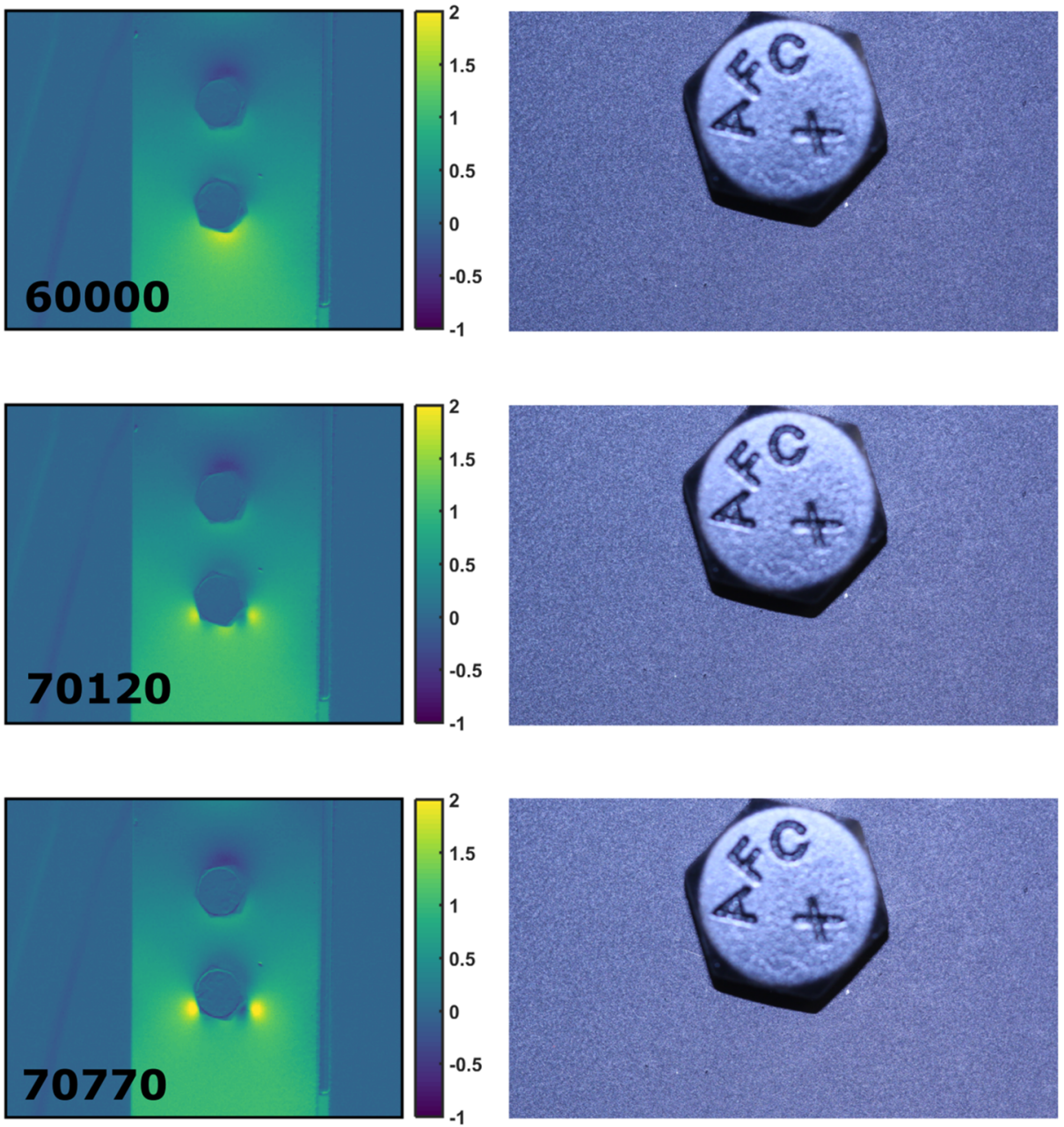

Fig. 8 Normalised TSA signal magnitude (left hand panels), visible light images (central panels) and magnified visible light images (right hand panels) for the complex bolted specimen, concentrating on crack

magnitude value is lower at 124,000 cycles than at 130,000 or later (similar effects can be seen for the other specimens in the supplementary information). The lower magnitudes, and more diffuse crack-tip stress distribution results in peaks in the difference data being consequently more diffuse.

Figure 7 shows that the results of the optical flow method processing of TSA data are very similar for specimens painted with matt black or primer paint. Rajic et al. [8]
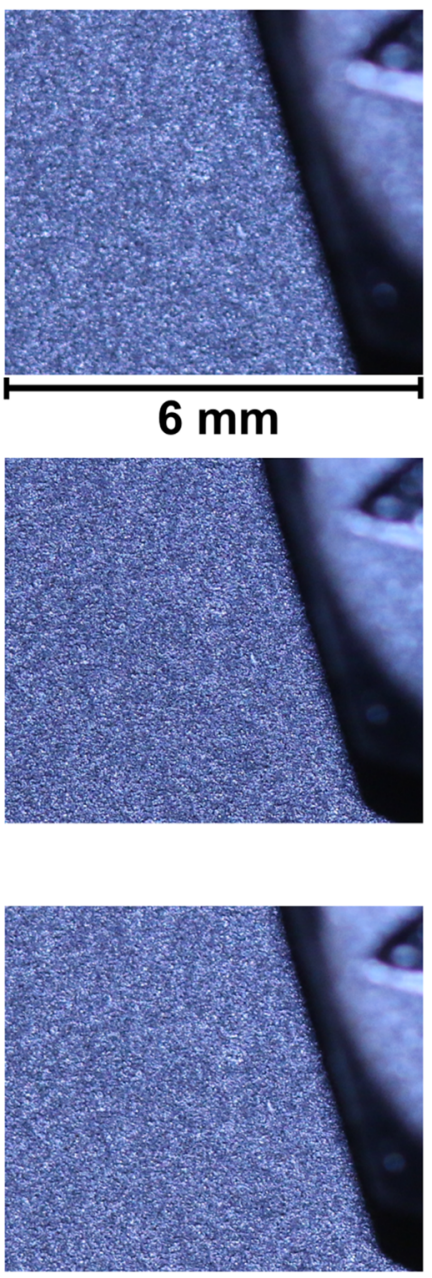

$6 \mathrm{~mm}$
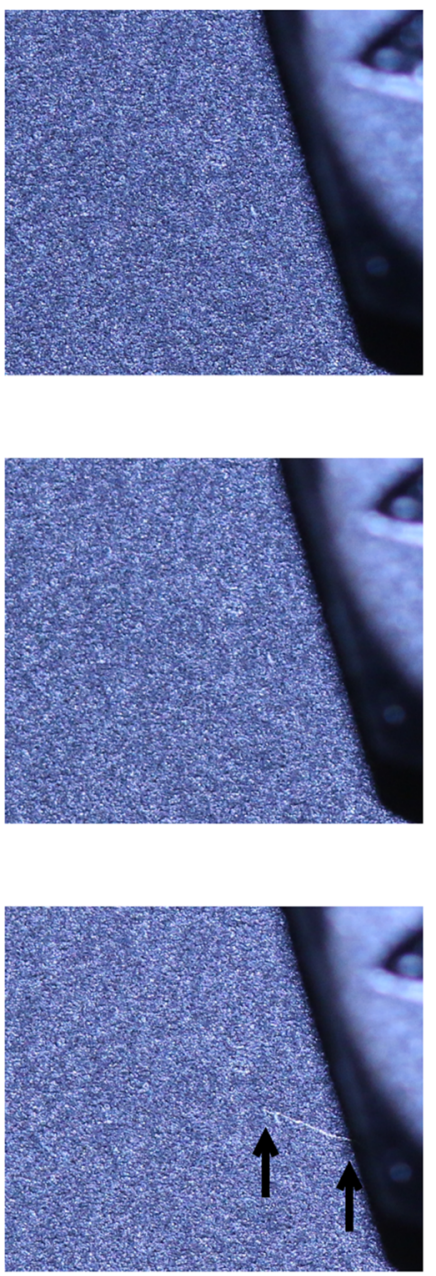

initiation on left hand side of bolt-head. Arrows indicate crack position on first observation in the visible light images

have previously carried out a comparison of TSA on black and primer paint, showing that their results are equivalent, and that adding matt black paint on top of primer paint increases the attenuation of the signal. The findings reported here, combined with those of [8], indicate that TSA and the optical flow method are of particular interest for crack tracking in aerospace applications, where no further 

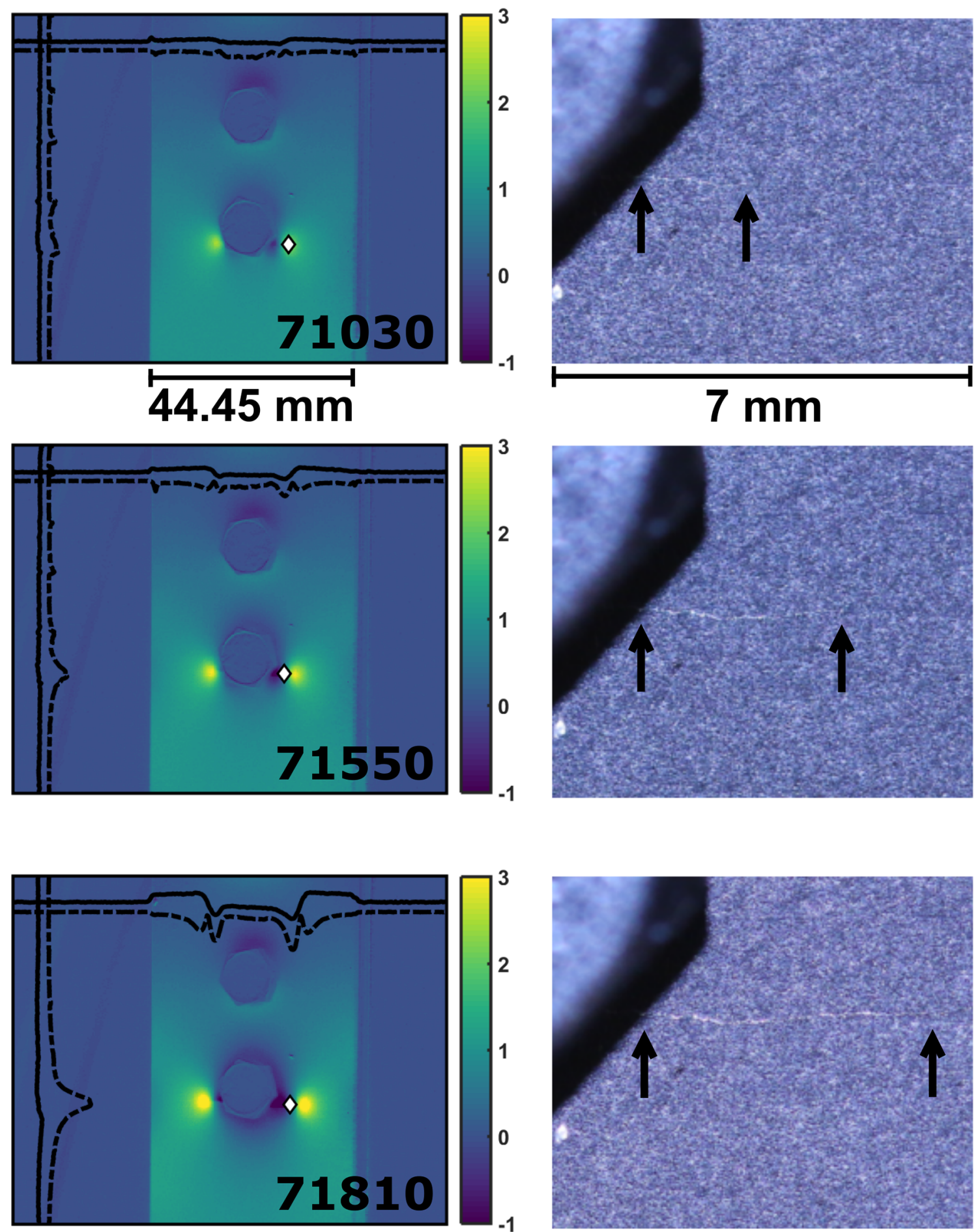

Fig. 9 Optical flow method results (diamonds) superimposed on the normalised TSA signal magnitude data at given cycle numbers (left hand panels). Same dataset as in Fig. 8, but here focussing on crack on the

right hand-side of the bolt. Arrows indicate the crack position in the visible light images (right hand panels)

surface preparation would be required before implementation.

For a bolted specimen, the propagation of the crack can be followed with the optical flow method (as seen in Fig. 9). However, it can be seen in Fig. 8 that signal magnitudes repre-

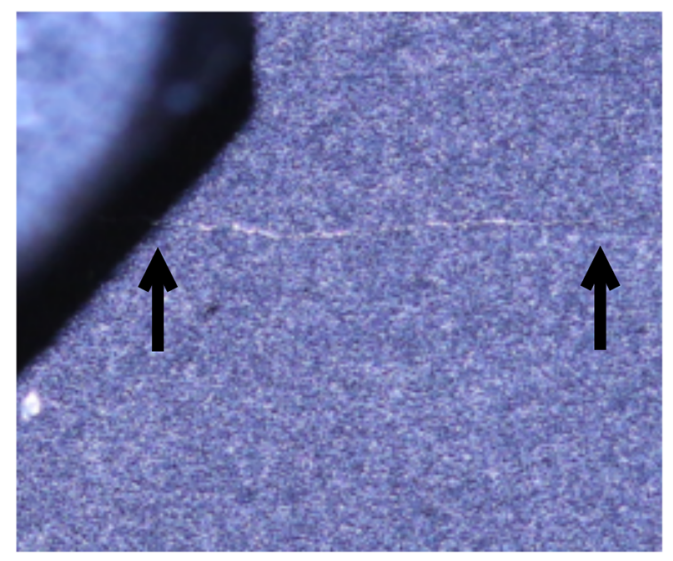

senting the crack-tip stress distribution have changed before the optical flow method locates them (Fig. 9). It is possible that the motion of the bolt during loading causes differences between TSA datasets, so overwhelming the changes due to initiation. This problem continues until the crack is 

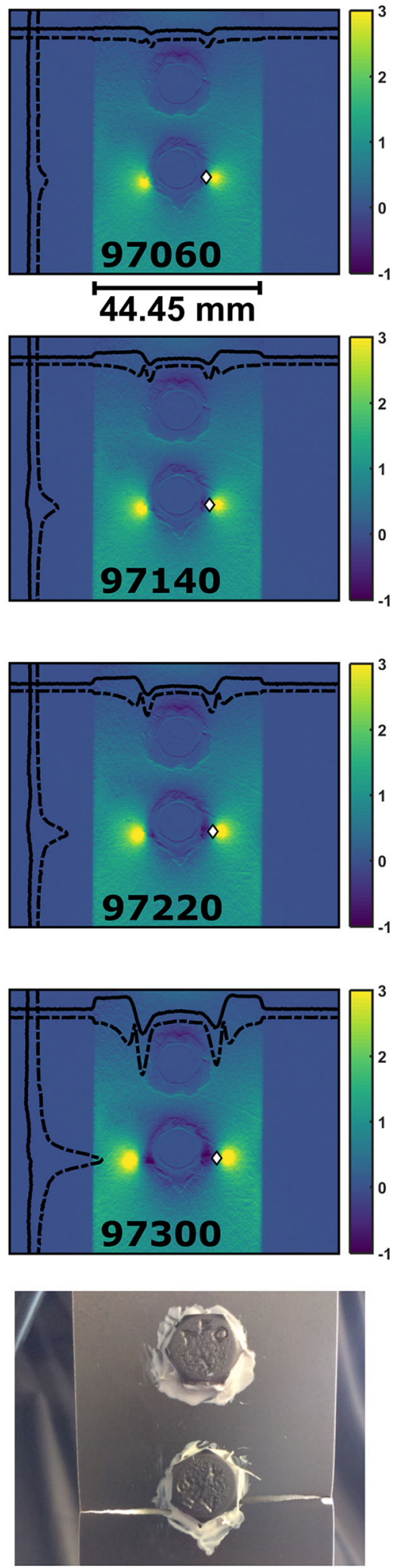

Fig. 10 Optical flow method results (diamonds) superimposed on the normalised TSA signal magnitude data at given cycle numbers for a bolted specimen with sealant around the bolt-heads. Final panel shows the specimen after failure far enough away from the bolt and the crack-tip stresses are higher, increasing the differences registered between datasets. Therefore, the optical flow method would require development for more complex geometries where the associated stress concentrations are not as simple. It is important to note here that although the optical flow method is limited in its ability to identify initiation, it still indicates crack development when visual observations are difficult, and that the visible light images shown here are at a higher magnification than an inspector would be working with in an industrial setting.

Where sealant is present around a bolt (Fig. 10), TSA data do not represent the stresses present in the metal under the sealant, as it acts as a thermal insulator. The detection of a crack using TSA is therefore delayed by the distance covered by the sealant, but this delay would also likely occur with other visual observation methods.

Here, rather than interrogating the phase data for each dataset to determine the crack-tip position [10-12], the pixel position of the crack-tip is defined by the movement of the maximum value of the crack-tip stress, as determined by the peak difference between two datasets. This simplification means the 'crack-tip' defined here is not necessarily coincident with the 'real' crack-tip; however, as can be seen in the comparison of plotted position on the final crack geometry (Fig. 7), this definition locates the crack position within a suitable margin. As the difference data reports an 'average' position between the two stress peaks in the TSA data, if the two datasets are too far apart in relation to the crack growth-rate and crack length, the peak value will have moved significantly between them, and the position indicated by the optical flow will be less accurate. This problem can be mitigated by quasi-continuous data collection, which could be interrogated in situ.

By definition, the spatial resolution of the optical flow method is limited by the pixel resolution of the image, a function of the sensor resolution and the size of the observed field of view (controlled by stand-off distance of the camera and the lens set-up of the camera). Here the resolution ranges from approximately $0.1-0.5 \mathrm{~mm}$ per pixel, but with larger fields of view, which may be necessary when considering larger industrial structures, the resolution will be lower. One possibility to improve this accuracy over large structures is to consider a scanning methodology.

Currently, including boundaries, such as the edge of the specimen, the hole in the specimen or the bolt-head in the interrogated region of interest can result in false positives for crack location, particularly before or at initiation. This effect is likely due to movement of the specimen resulting in peaks in the mean and standard deviation, as can be seen at the edges of the specimen in Fig. 5 at 140,000 cycles, and to a lesser degree at 124,000 cycles, and also at the bolt-head in Fig. 9. In this work, this problem has been mitigated by careful choice of the region of interest, resulting in the crack- 
tip position being located within $1 \mathrm{~mm}$ of the final crack morphology. In future applications this could also be controlled by evaluating the choice of threshold for significant changes, or implementing a method of motion compensation [25]. These options will likely become more important when considering more complex applications.

From Specimen 5 in Fig. 6b, it can also be seen that the optical flow method has misidentified the crack location at 9500 cycles, as the crack length is reported as decreasing. This misidentification is likely due to a combination of fast crack growth just before failure, and significant increases in stress at the crack-tip. This effect could be solved by a higher rate of data collection.

In order for TSA data to be collected, certain loading criteria must be met, such that the stresses induced are high enough to be detected. The stress sensitivity depends on the material and the surrounding temperature, but compares favourably to that of strain gauges [5]. If the loading forces are high enough to result in the initiation and propagation of fatigue cracks, then the conditions will be such that stresses are visible in TSA data. However, when loading is at very low frequencies, TSA is no longer appropriate, as the thermoelastic response is no longer adiabatic [5]. When in situ TSA data collection is not possible during the existing or service loads, an alternative approach would be to introduce excitation during inspection periods through other methods (e.g. vibration loading [6]).

In conventional TSA, errors can be introduced as a consequence of loading frequency, paint thickness and specimen motion. These errors will be constant or only vary slowly with time during a fatigue test; and, hence the use of differential datasets (i.e. the difference between two sequential datasets of TSA signal magnitude) eliminates the effects of these errors. However, one disadvantage of the optical flow method is that it will only locate a crack if that crack is propagating. Therefore, methods which interrogate the TSA data directly, rather than the difference between TSA datasets could be more appropriate in certain applications. The optical flow method presented here is best applied for early indication of a naturally initiating crack, determining propagation direction, and determining when propagation reaches a critical point indicating that failure is imminent, whilst, for example the method of Rajic and Brooks [12] allows for more precise locating of the crack-tip position.

To fully automate this optical flow method, user input must be removed. This change would require automated selection of the region of interest, for example by selecting an area around the peak stress, similar to the selection method of Rajic and Brooks [12]. This selection would then also allow the area external to the initial crack to be interrogated separately to locate further cracks as they develop. Currently the user determines at what level of precision the optical flow method runs, which could be fixed to a certain level of uncer- tainty determined by the standard deviation of the difference image.

It would be interesting, particularly in industrial contexts, to have the functionality to monitor the crack in real time. As it is presented here, this optical flow method is implemented ex situ with the processing implemented in MATLAB; however, it should be reasonably simple to incorporate into a TSA software programme. The computer processing time would be dependent on the number of datasets interrogated and the size of those datasets, so the capability of processing large amounts of data in a short time period would be necessary.

Previous studies which automate processing of TSA data have used pre-cracked notched specimens such that a crack is artificially initiated and is evident in the TSA data before propagation occurs [11, 12]. The technique of Ancona et al. [11] also requires previous knowledge of the position of the crack-tip after pre-cracking, as well as a calibration constant, calculated for each experiment, which is dependent on the material and experimental set-up. Further, in order to avoid problems with specimen motion, Rajic and Brooks [12] paused loading to collect TSA data.

The optical flow method proposed here therefore has certain advantages, as it can be applied to situations both where cracks initiate naturally, and where there is an already initiated crack. It can be applied with no prior knowledge of the material properties, and to TSA data output from any system. The interrogation time of the TSA data (on the order of $10 \mathrm{~s}$, including TSA signal post-processing) is also faster than the technique of Ancona et al. [11] (on the order of $2 \mathrm{~min}$ ) and it is not necessary to stop loading to collect TSA data. Therefore, this optical flow method has the potential to locate sub-mm length cracks as they initiate, and incorporating the optical flow method in TSA software would allow fast-propagating cracks to be tracked in real-time in industrial applications.

\section{Conclusions}

This study has shown that processing of thermoelastic stress analysis data with an algorithm which uses the concept of optical flow allows observation of the natural initiation of cracks at sub-mm lengths, before that crack is observable by visual inspection. Tracking the propagation of cracks has been achieved in specimens with simple and complex geometries. Crack-tip positions mapped using this method were within $\pm 1 \mathrm{~mm}$ of the real crack position, as measured after failure of the specimen. In bolted specimens, a particular advantage of this method is that the TSA data will locate the initiation of a crack before the crack-tip becomes visible from underneath the bolt-head. The presence of sealant around the bolt-head delays this detection, but only by the extent of the sealant cover, so it would be expected that the same delay would be present for visual inspection. 
The paint finish on the observed material is important for TSA to work effectively, the results presented here agree with those of Rajic et al. [8] that aircraft primer paint gives equivalent results to matt black paint. Therefore, it would not be necessary to re-paint parts during testing, so increasing the applicability and time-effectiveness of TSA in certain industrial contexts. This work could be expanded to include the optical flow method in TSA software to allow real-time, in situ location of cracks before they are visible through conventional techniques.

Acknowledgements The INSTRUCTIVE project is funded by the EU Framework Programme for Research and Innovation Horizon 2020, Clean Sky 2, Project Number 686777. Helpful assistance was provided by the project topic managers, Niels Strohmaier (Airbus Operations $\mathrm{GmbH}$ ) and Eszter Szigeti (Airbus Operations Ltd). We thank two anonymous reviewers for helpful comments on the manuscript.

Data Availability Statement The datasets generated and analysed during the current study are available from the University of Liverpool Data Catalogue, http://datacat.liverpool.ac.uk/id/eprint/468, DOI: https://doi.org/10.17638/datacat.liverpool.ac.uk/468.

Open Access This article is distributed under the terms of the Creative Commons Attribution 4.0 International License (http://creativecomm ons.org/licenses/by/4.0/), which permits unrestricted use, distribution, and reproduction in any medium, provided you give appropriate credit to the original author(s) and the source, provide a link to the Creative Commons license, and indicate if changes were made.

\section{References}

1. Sakagami, T.: Remote nondestructive evaluation technique using infrared thermography for fatigue cracks in steel bridges. Fatigue Fract. Eng. Mater. Struct. 38, 755-779 (2015). https://doi.org/10. 1111/ffe. 12302

2. Rastogi, P., Hack, E. (eds.): Optical Methods for Solid Mechanics. Wiley-VCH, Weinheim (2012)

3. Epstein, J.S. (ed.): Experimental Techniques in Fracture. VCH, Weinheim (1993)

4. Patterson, E.A., Feligiotti, M., Hack, E.: On the integration of validation, quality assurance and non-destructive evaluation. J. Strain Anal. Eng. Des. 48, 48-58 (2013). https://doi.org/10.1177/ 0309324712444681

5. Greene, R.J., Patterson, E.A., Rowlands, R.E.: Thermoelastic stress analysis. In: Sharpe, W.N. (ed.) Springer Handbook of Experimental Solid Mechanics, pp. 743-767. Springer, New York (2008)

6. Tighe, R.C., Howell, G.P., Tyler, J.P., Lormor, S., Dulieu-Barton, J.M.: Stress based non-destructive evaluation using thermographic approaches: from laboratory trials to on-site assessment. NDT E Int. 84, 76-88 (2016). https://doi.org/10.1016/j.ndteint.2016.08. 005

7. Robinson, A.F., Dulieu-Barton, J.M., Quinn, S., Burguete, R.L.: Paint coating characterization for thermoelastic stress analysis of metallic. Meas. Sci. Techol. (2010). https://doi.org/10.1088/09570233/21/8/085502

8. Rajic, N., Street, N., Brooks, C., Galea, S.: Full field stress measurement for in situ structural health monitoring of airframe components and repairs. In: 7th European Workshop on Structural Health Monitoring (2014)
9. Dulieu-Barton, J.M.: Introduction to thermoelastic stress analysis. Strain 35(2), 35-39 (1999)

10. Diaz, F.A., Patterson, E.A., Tomlinson, R.A., Yates, J.R.: Measuring stress intensity factors during fatigue crack growth using thermoelasticity. Fatigue Fract. Eng. Mater. Struct. 27, 571-583 (2004). https://doi.org/10.1111/j.1460-2695.2004.00782.x

11. Ancona, F., Palumbo, D., De Finis, R., Demelio, G.P., Galietti, U.: Automatic procedure for evaluating the Paris Law of martensitic and austenitic stainless steels by means of thermal methods. Eng. Fract. Mech. 163, 206-219 (2016). https://doi.org/10.1016/j. engfracmech.2016.06.016

12. Rajic, N., Brooks, C.: Automated crack detection and crack growth rate measurement using thermoelasticity. Procedia Eng. 188, 463-470 (2017). https://doi.org/10.1016/j.proeng.2017.04.509

13. Hack, E., Fruehmann, R.K., Roos, R., Feligiotti, M., Schuetz, P., Tyler, J.P., Dulieu-Barton, J.M.: Flaw and damage assessment in torsionally loaded CFRP cylinders using experimental and numerical methods. Compos. Struct. 132, 109-121 (2015). https://doi. org/10.1016/j.compstruct.2015.05.025

14. Fruehmann, R.K., Dulieu-Barton, J.M., Quinn, S.: Thermoelastic stress and damage analysis using transient loading. Exp. Mech. 50, 1075-1086 (2010). https://doi.org/10.1007/s11340-009-9295-9

15. Rajic, N., Galea, S.: Thermoelastic stress analysis and structural health monitoring: an emerging nexus. Struct. Heal. Monit. 14, 57-72 (2015). https://doi.org/10.1177/1475921714548936

16. Sakagami, T., Kubo, S., Tamura, E., Nishimura, T.: Identification of plastic-zone based on double frequency lock in thermographic temperature measurement. In: International Conference on Fracture ICF11 (2005)

17. Paynter, R.J.H., Dutton, A.G.: The use of a second harmonic correlation to detect damage in composite structures using thermoelastic stress measurements. Strain 39, 73-78 (2003). https://doi.org/10. 1046/j.1475-1305.2003.00056.x

18. Backman, D., Cowal, C., Patterson, E.A.: Analysis of the effects of cold expansion of holes using thermoelasticity and image correlation. Fatigue Fract. Eng. Mater. Struct. 33, 859-870 (2010). https:// doi.org/10.1111/j.1460-2695.2010.01472.x

19. Rajic, N., Rowlands, D.: Thermoelastic stress analysis with a compact low-cost microbolometer system. Quant. Infrared Thermogr. J. 10, 135-158 (2013). https://doi.org/10.1080/17686733. 2013.800688

20. Rajic, N., Street, N.: A performance comparison between cooled and uncooled infrared detectors for thermoelastic stress analysis. Quant. Infrared Thermogr. J. 11, 207-221 (2014). https://doi.org/ 10.1080/17686733.2014.962835

21. BSI Standards Publication Mechanical joining-Destructive testing of joints-Specimen dimensions and test procedure for tensile shear testing of single joints. BS EN ISO 12996:2013. (2013)

22. Eurocode 9: Design of aluminium structures - Part 1-1: General structural rules. BS EN 1999-1-1:2007 + A 1 :2009. (2009)

23. Díaz, F.A., Yates, J.R., Patterson, E.A.: Some improvements in the analysis of fatigue cracks using thermoelasticity. Int. J. Fatigue 26, 365-376 (2004). https://doi.org/10.1016/j.ijfatigue.2003.08.018

24. Horn, B.K.P., Schunck, B.G.: Determining Optical Flow. Artif. Intell. 17, 185-203 (1981)

25. Sakagami, T., Yamaguchi, N., Kubo, S., Nishimura, T.: A new full-field motion compensation technique for infrared stress measurement using digital image correlation. J. Strain Anal. Eng. Des. 43, 539-549 (2008). https://doi.org/10.1243/03093247JSA360 